\title{
Treatment of multiple traumatized anterior teeth associated with an alveolar bone fracture in a 20-year-old patient: a 3-year follow up
}

\author{
Vicente Faus-Matoses ${ }^{1}$, María Martínez-Viñarta ${ }^{1}$, Teresa Alegre-Domingo ${ }^{1}$, Ignacio Faus-Matoses ${ }^{1}$, Vicente \\ J. Faus-Llácer ${ }^{3}$ \\ ${ }^{1}$ DDS, MSc. Master of Restorative Dentristy and Endodontics, Department of Stomatology, Medicine and Dental School, Valen- \\ cia University, Spain \\ ${ }^{2}$ DDS, MSc. Master in Prosthetic Dentistry, Department of Stomatology, Medicine and Dental School, Valencia University, Spain \\ ${ }^{3}$ MD, DDS, MSc, PhD. Master of Restorative Dentristy and Endodontics, Department of Stomatology, Medicine and Dental \\ School, Valencia University, Spain
}

Correspondence:

C/ Gascó Oliag n $n^{\circ} .1$

Unidad de Patología y Terapéutica Dental

Clínica Odontológica,

46010 Valencia, Spain

mariamartinezvinarta@gmail.com

Received:11/11/2013

Accepted: $01 / 05 / 2014$

Faus-Matoses V, Martínez-Viñarta M, Alegre-Domingo T, Faus-Matoses I, Faus-Llácer VJ. Treatment of multiple traumatized anterior teeth associated with an alveolar bone fracture in a 20-year-old patient: a 3-year follow up. J Clin Exp Dent. 2014;6(4):e425-9.

http://www.medicinaoral.com/odo/volumenes/v6i4/jcedv6i4p425.pdf

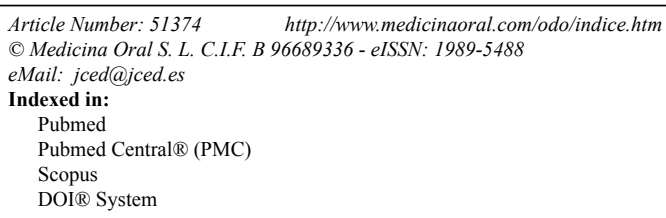

\begin{abstract}
Intrusive luxation is a type of recognizable luxation injury represented by a deeper axial displacement of the tooth toward the alveolar bone. Treatment strategies include waiting for the tooth to return to its position, immediate surgical repositioning, and repositioning through dental traction by orthodontic devices. The aim of this case report was to present the management of severe dental trauma and later restoration following IADT. A 20-year-old patient was presented after fainting at home four hours before, resulting in a dento-alveolar trauma. Clinical examinations revealed a traumatic intrusion, in 1.2, 1.1 and 2.1, uncomplicated crown fractures in 1.1 and 2.1 and a complicated crown-root fracture in 2.2. The diagnosis was confirmed with CBCT. Following IADT protocol, the emergency treatment consisted of the surgical repositioning and semi-rigid splinting using orthodontic wire-composite, replacing the buccal bone plate, and postoperative instructions to the patient regarding oral hygiene. After 2 weeks the root canal treated and filled with fiberglass posts in 1.2, 1.1, 2.1 and 2.2. Splint was removed after 4 weeks and the IADT reassessment protocol followed, with revisions at 6-8 weeks, 6 months, a year and annual reviews for 5 years. A year after the treatment, the traumatized teeth were restored with minimally invasive preparations of feldspathic ceramic. Esthetics and function were recorded with a 3-year follow-up period.
\end{abstract}

Key words: Intrusive luxation, dental trauma, crown-root fracture, dento-alveolar trauma, permanent tooth, CBCT.

\section{Introduction}

Intrusive luxation is a type of recognizable luxation injury characterized by a deeper axial displacement of the tooth toward the alveolar bone (1), representing 0.3$1.9 \%$ of dental trauma in permanent teeth (2). The highest incidence occurs in boys between 6-12 years old (3) which makes treatment of these injuries difficult (4). The upper central incisors are commonly the most affected teeth [93.3\%]. Predominant etiologic factors are falls at home [60\%], followed by bicycle injuries $(2,5)$.

Severe dental traumas can involve numerous injuries, including intrusion [33.5\%], an associated crown fracture intrusion [60.5\%], or a combination of intrusion and coronal or root fractures [6\%] (2). In most cases it affects only one tooth [46.3\%], followed by two teeth [32.4\%] and three or more teeth [21.3\%] (2). Most of the intruded 
teeth are displaced $2-8 \mathrm{~mm}$ into the alveolar bone (2), with infrequent intrusions occurring over $6 \mathrm{~mm}$ (5).

Three types of treatment have been proposed for traumatic intrusions in permanent teeth: watchful waiting with passive repositioning [41.7\%], immediate surgical repositioning [58.3\%] (5) or orthodontic traction. The indications for treatment strategy depend on the stage of root development, the severity of the intrusion, the presence of alveolar fracture or the number of teeth involved in multiple intrusions. The focus must be on the elimination or attenuation of the injury to avoid future complications (4,6-8).

The aim of this case report was to present the management of severe dental trauma in a 20-year-old man and later restoration following the protocol of International Association for Dental Traumatology [IADT].

\section{Case Report}

A 20-year-old male was referred to the Department of Stomatology, Master of Restorative Dentistry and Endodontics, Medicine and Dental School, Valencia University, Spain, after fainting at home four hours before, resulting in a dento-alveolar trauma. His medical history included absence seizures as a child and the use of an orthodontic device for eight months before the trauma.

Intraoral examination revealed a traumatic intrusion, greater than $7 \mathrm{~mm}$, in $1.2,1.1$ and 2.1 , uncomplicated crown fractures in 1.1 and 2.1 and a complicated crownroot fracture in 2.2 (Fig.1). The patient also suffered lower lip and chin laceration (Fig. 1). The diagnosis was confirmed with CBCT radiographic examination, which showed a fracture of the vestibular cortical bone (Fig. 2).

Following IADT protocol, the emergency treatment consisted, under local anesthesia [epinephrine 1:100,000], on the surgical repositioning, removal the fragment of 2.2 with forceps and semi-rigid splinting using orthodontic wire-composite (Fig. 3); replacing the buccal bone plate, and postoperative instructions to the patient regarding oral hygiene using chlorhexidine $0.1 \%$ rinses for 2 weeks. No antibiotic coverage was administered, because there were no significant differences in healing if antibiotics are used or not (6).

After 2 weeks the stitches were removed and the root canal treated was performed in 1.2, 1.1, 2.1 and 2.2 using rotary instruments [ProTaper Universal, Dentsply Maillefer, Ballaigues, Switzerland] and sealed with thermoplasticized gutta-percha [Thermafil, Dentsply Maillefer, Ballaigues, Switzerland]. The teeth were then restored with fiberglass posts [Normopost, Normon, Biolonen, Saronno, Italy] (Fig. 3). In addition, in 2.2 a palatal gingivectomy and ostectomy was performed to prevent the invasion of the periodontal space of the future restoration. Splint was removed after 4 weeks (Fig. 3) and the IADT reassessment protocol followed with revisions at 6-8 weeks, 6 months, a year and annual reviews for 5 years (9). A year after the treatment, the traumatized teeth were restored with minimally invasive preparations of feldspathic ceramic [Creation Willi Geller International $\mathrm{GmbH}$, Meiningen, Austria] (Fig. 3).

\section{Discussion}

Dentoalveolar trauma involving multiple teeth are rare (10), so its treatment remains a challenge for professionals. This study aims to report a multiple trauma with severe intrusions of three teeth, uncomplicated crown fractures, complicated crown-root fracture, and vestibular cortical bone fracture.

Intrusive dislocations are diagnosed based on clinical signs and symptoms, such as lack of occlusal alignment,
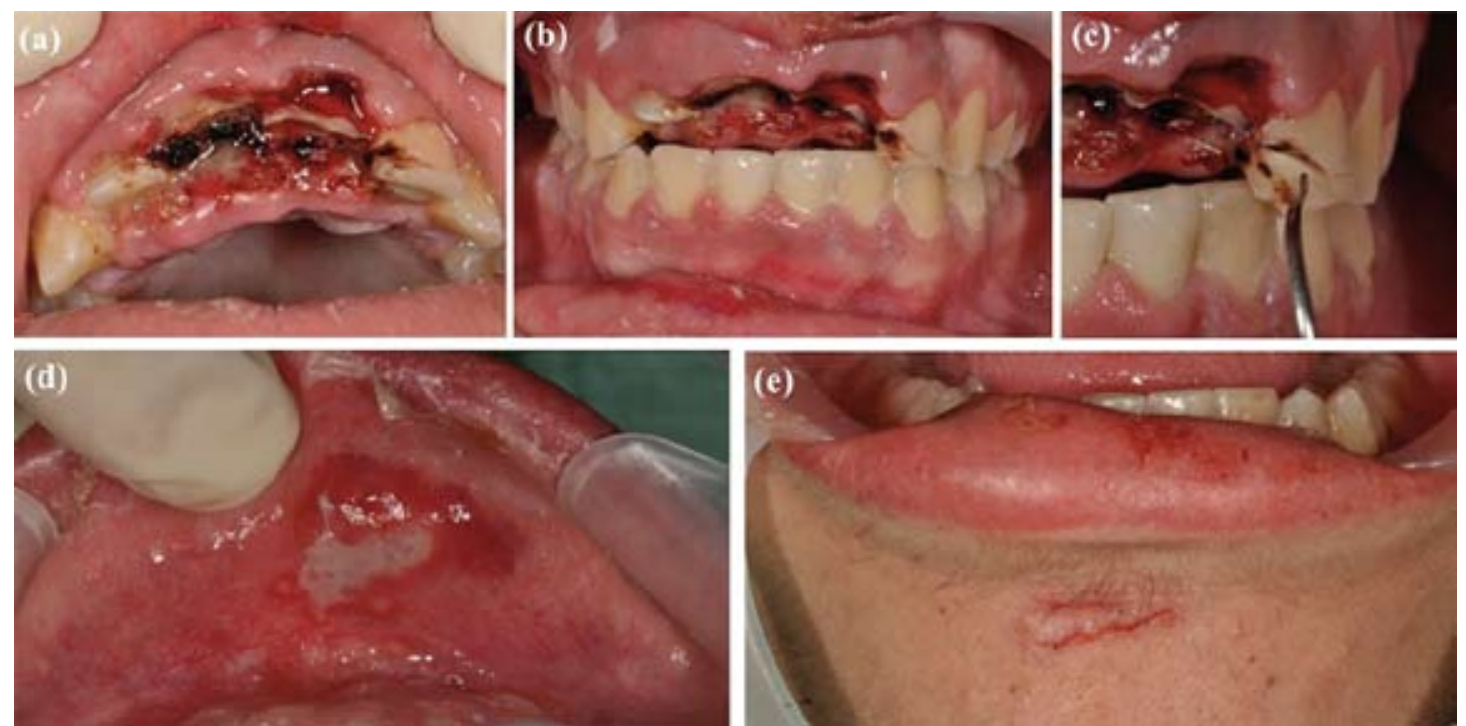

Fig. 1. (a) Oclusal view, traumatic intrusion in 1.2, 1.1 and 2.1. (b) Vestibular view, traumatic intrusion in 1.2, 1.1 and 2.1. (c) Complicated crown-root fracture in 2.2. (d) Lower lip laceration. (e) Chin lateration. 

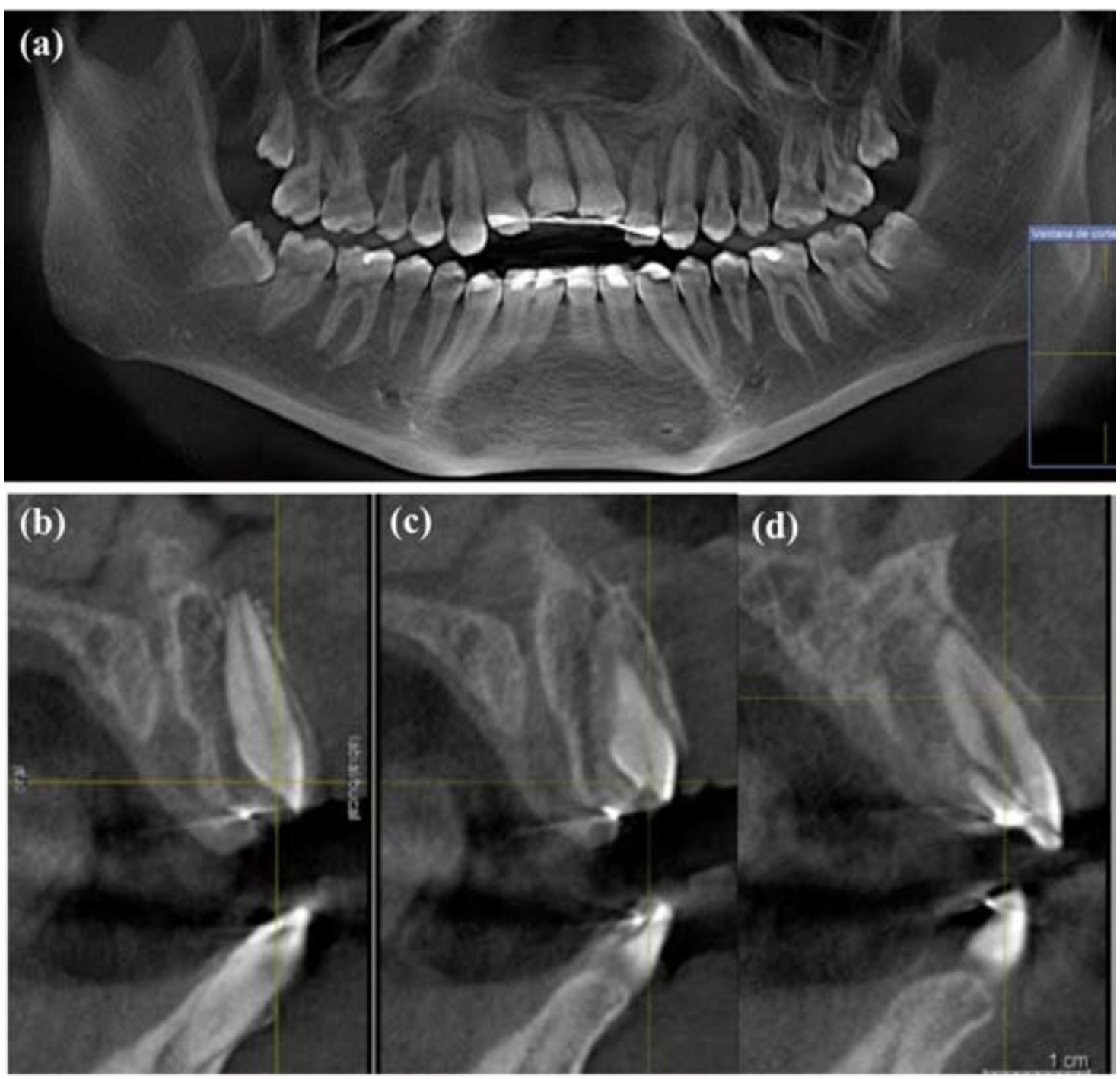

Fig. 2. (a) The initial orthopantomography. (b) CBCT radiographic examination, showed an alveolar bone fracture and traumatic luxation in 1.1. (c) CBCT radiographic examination showed a fracture of the vestibular cortical bone. (d) CBCT radiographic examination showed a complicated crown-root fracture in 2.2.

bleeding and a dry thud sound when tapped (4). In this study, radiographic examination revealed that the periodontal ligament space had disappeared as had the height difference between the apexes of the traumatized teeth and their counterparts.

The incorporation of CBCT has significantly improved the ability to accurately diagnose traumatic injuries (11). The use in this study of CBCT images of the vestibular cortical bone provided high diagnostic accuracy that resulted in a good treatment strategy.

Three types of treatments for intruded teeth are proposed without reaching a consensus for an optimal alternative: passive spontaneous reposition, surgical reposition and orthodontic reposition (5). The treatment strategy will depend on the development stage of the root apex, the severity of the intrusion, the presence of alveolar fractures or multiple intrusions, and should be focused on the elimination or attenuation of post-injury complications

\section{$(2,4,6-8)$.}

The correct treatment for intruded teeth with complete root formation and intrusion greater than $7 \mathrm{~mm}$ is, according to Diangelis et al. (9), a surgical extrusion and stabilizing the tooth using a flexible splint for 4-8 weeks. However, Andresen et al. (6) conducted a study of 140 intruded teeth and evaluated their healing according to the type of splinting and found no significant differences. In this study, a semi-rigid wire-composite splint was used as suggested by Berthold et al. (12), since it is also suitable for cortical bone fractures. To prevent pulp necrosis, root canal treatment of the teeth intruded is recommended after 2-3 weeks of the surgical extrusion (6).

The emergency treatment for complicated crown-root fractures, according to Diangelis et al. (9), is the temporary stabilization of the lost fragment with the adjacent tooth. Subsequently, the fragment should be removed, 

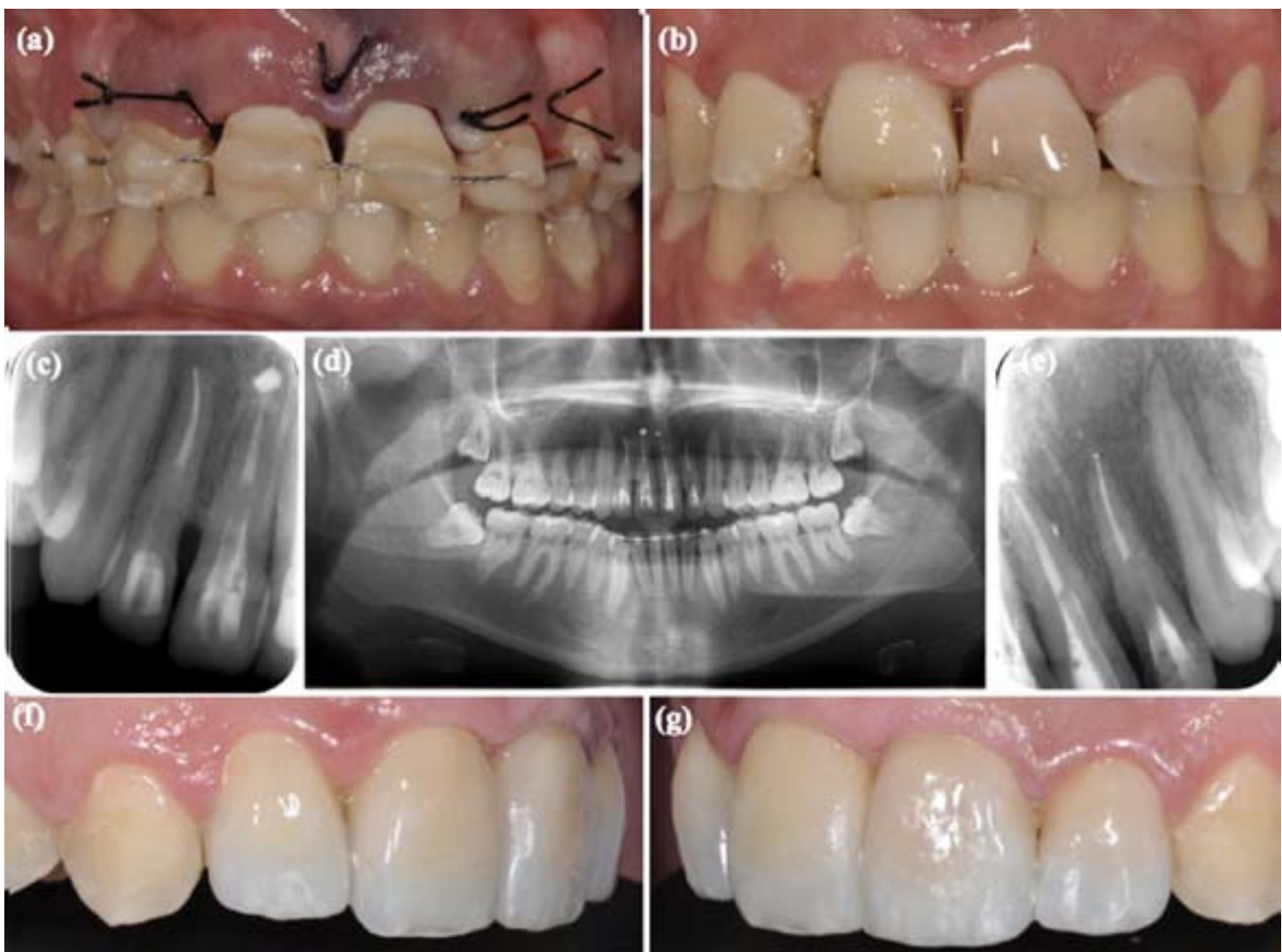

Fig. 3. (a) Surgical repositioning and semi-rigid splinting using orthodontic wire-composite. (b) Splint was removed. (c) Root canal treatment and filling with glass fiber posts in 1.1 and 1.2. (d) Orthopantomography, 2 weeks follow-up. (e) Root canal treatment and filling with fiberglass posts in 2.1 and 2.2. (f) Lateral left view, the traumatized teeth were restored with minimally invasive preparations of feldspathic ceramic. (g) Lateral right view.

the root canals treated and coronal restoration made with fiberglass post. This procedure must be preceded by a gingivectomy and sometimes by an osteoplasty and ostectomy. This type of treatment is only suitable for crown-root fractures with subgingival palatal extension, as in this case report in which there was a complicated crown-root fracture of 2.2 with subgingival palatal extension.

Successful healing following injury depends mainly on the development stage of the root. There is less risk of complications in cases of incomplete root formation. This is probably because of a softer bone surrounding the teeth, which requires smaller penetrations for periodontal ligament injuries. Crown fractures [with exposed dentin] had a negative influence on pulp healing. The extent of the intrusion was partly influenced by the degree of intrusion being higher than $7 \mathrm{~mm}$. Finally, the adjacent intruded teeth had a negative influence on the healing of the marginal bone between the intruded teeth $(4,13)$.

In conclusion, severe dental trauma involving three or more teeth are a challenge for dentists. The teeth involved in the trauma should be regularly monitored in order to detect and treat possible complications arising as a result. After the three years control period, it was conclu- ded that following the IADT protocol proved successful in preserving the dental health, and satisfying both the aesthetic and functional wishes of the patient. Understanding current treatment guidelines will improve the care of dental trauma in general and emergency care, in particular.

\section{References}

1. Luna AH, Moreira RW, de Moraes M. Traumatic intrusion of maxillary permanent incisors into the nasal cavity: report of a case. Dent Traumatol. 2008;24:244-7.

2. Andreasen JO, Bakland LK, Matras RC, Andreasen FM. Traumatic intrusion of permanent teeth. Part 1. An epidemiological study of 216 intruded permanent teeth. Dent Traumatol. 2006;22:83-9.

3. Faus-Damiá M, Alegre-Domingo T, Faus-Matoses I, Faus-Matoses V, Faus-Llácer VJ. Traumatic dental injuries among schoolchildren in Valencia, Spain. Med Oral Pathol Oral Cir Bucal. 2011;1:e292-5.

4. Andresen JO, Bakland LK, Andresen FM. Traumatic intrusion of permanent teeth. Part 2. A clinical study of the effect of preinjury and injury factors, such as sex, age, stage of root development, tooth location, and extent of injury including number of intruded teeth on 140 intruded permanent teeth. Dental Traumatol. 2006;22:90-8.

5. Neto JJ, Gondim JO, de Carvalho FM, Giro EM. Longitudinal clinical and radiographic evaluation of severely intruded permanent incisors in a pediatric population. Dent Traumatol. 2009;25:510-4.

6. Andreasen JO, Bakland LK, Andreasen FM. Traumatic intrusion of permanent teeth. Part 3. A clinical estudy of the effect of treatment variables such as treatement delay, method of repositioning, type of splint, length of splinting and antibiotics on 140 teeth. Dent Traumatol. 
2006;22:99-111.

7. Faria G, Silva RA, Fiori-Junior M, Nelson-Filho P. Re- eruption of traumatically intruded mature permanent incisor: case report. Dent Traumatol. 2004;20:229-32.

8. Flores MT, Andersson L, Andreasen JO, Bakland LK, Malmgren B, Barnett F, Bourguignon C, DiAngelis A, Hicks L, Sigurdsson A, Trope M, Tsukiboshi M, von Arx T. Guidelines for the management of traumatic injuries. I. Fractures and luxations of permanent teeth. Dent Traumatol. 2007;23:66-71.

9. Diangelis AJ, Andreasen JO, Ebeleseder KA, Kenny DJ, Trope M, Sigurdsson A, Andersson L, Bourguignon C, Flores MT, Hicks ML, Lenzi AR, Malmgren B, Moule AJ, Pohl Y, Tsukiboshi M. International Association of Dental Traumatology guidelines for the management of traumatic dental injuries: 1. Fractures and luxations of permanent teeth. Dent Traumatol. 2012;28:2-12.

10. Koyuturk AE, Kusgoz A. Multiple dentoalveolar traumatic injury: a case report (3 years follow up). Dent Traumatol. 2008;24:e16-19.

11. Scarfe WC. Imaging of maxillofacial trauma: evolutions and emerging revolutions. Oral Surg Oral Med Oral Pathol Oral Radiol Endod. 2005;100(Suppl. 2):S75-96.

12. Berthold C, Thaler A, Petschelt A. Rigidity of commonly used dental trauma splints. Dent Traumatol. 2009;25:248-55.

13. Traebert J, Peres MA, Blank V, Boell Rda S, Pietruza JA. Prevalence of traumatic dental injury and associated factors among 12year-old school children in Florianopolis, Brazil. Dent Traumatol. 2003;19:15-8.

\section{Conflict of Interest}

The authors declare that they have no conflict of interest. 